ANNALES

POLONICI MATHEMATICI

$93.2(2008)$

\title{
On the Kuratowski convergence of analytic sets
}

\author{
by Maciej P. Denkowski and Rafae Pierzchata (Kraków)
}

\begin{abstract}
We discuss some conditions which guarantee that the Kuratowski limit of a sequence of analytic sets is a Nash set.
\end{abstract}

1. Preliminaries. Let $X \subset \mathbb{C}^{n}$ be a locally closed set. Let $\mathcal{F}_{X}$ denote the family of all closed subsets of $X$. In $\mathcal{F}_{X}$ we have the topology generated by the sets

$$
\mathcal{U}(S, K)=\left\{F \in \mathcal{F}_{X}: F \cap K=\emptyset, F \cap U \neq \emptyset \text { for } U \in S\right\},
$$

where $K \subset X$ is compact and $S$ is a finite family of open subsets of $X$. This topology has the following properties:

(1) $\mathcal{F}_{X}$ is a metrizable compact space. The convergence in this topology is called the Kuratowski convergence.

(2) $F_{\nu} \rightarrow F$ in $\mathcal{F}_{X}$ if and only if for any $x \in F$ there exist $x_{\nu} \in F_{\nu}$ such that $x_{\nu} \rightarrow x$ and for every compact subset $K \subset X \backslash F$ we have $K \cap F_{\nu}=\emptyset$ for all sufficiently large $\nu$.

(3) The map $\mathcal{F}_{X} \times \mathcal{F}_{X} \ni(A, B) \mapsto A \cup B \in \mathcal{F}_{X}$ is continuous.

(4) If $Y \subset X$ is open, then the map $\mathcal{F}_{X} \ni F \mapsto F \cap Y \in \mathcal{F}_{Y}$ is continuous.

For (1) and (2), see [10, Lemmas 1 and 2]. The remaining two properties follow easily from (2).

REMARK 1.1. Let $\Omega \subset \mathbb{C}^{n}$ be open. If $A_{\nu} \rightarrow A$ in $\mathcal{F}_{\Omega}$ and $A_{\nu}$ are analytic and irreducible, then

(1) A need not be analytic.

(2) If $A$ is analytic, it need not be irreducible.

Proof. Put $A_{\nu}:=\left\{z^{p_{\nu}}=y^{q_{\nu}}\right\} \subset \mathbb{C}^{2}$, where $p_{\nu}, q_{\nu}$ are positive integers such that $\operatorname{gcd}\left(p_{\nu}, q_{\nu}\right)=1$ and $p_{\nu} / q_{\nu} \rightarrow \sqrt{2}$. Passing to a subsequence if necessary we may assume that $A_{\nu} \rightarrow A$. Suppose that $A$ is analytic. Note that $(0,0) \notin \operatorname{Int} A$. Clearly, if a convergent power series $f(z, y)=\sum a_{i j} z^{i} y^{j}$

2000 Mathematics Subject Classification: 14P15, 32B15.

Key words and phrases: Nash set, definable set, Kuratowski convergence. 
vanishes on the curve $\Gamma=\left\{\left(t, t^{\sqrt{2}}\right) \in \mathbb{R}^{2}: t \in[0, \varepsilon]\right\}$, where $\varepsilon>0$, then $f \equiv 0$ (because the map $\mathbb{N}^{2} \ni(i, j) \mapsto i+j \sqrt{2} \in \mathbb{R}$ is injective). Since $\Gamma \subset A$, we easily obtain a contradiction.

For the second part of the remark, we put $A_{\nu}:=\{z y=1 / \nu\} \subset \mathbb{C}^{2}$ and $A:=\{z y=0\}$.

Let $G_{k}^{\prime}\left(\mathbb{C}^{n}\right)$ denote the set of all $k$-dimensional affine subspaces of $\mathbb{C}^{n}$ (cf. [6]). Łojasiewicz defines a topology (we call it $\tau$ ) in $G_{k}^{\prime}\left(\mathbb{C}^{n}\right)$ in such a way that it is metrizable and has the following property: for any $L=z_{0}+\sum_{j=1}^{k} \mathbb{C} z_{j} \in$ $G_{k}^{\prime}\left(\mathbb{C}^{n}\right)$ and $L_{\nu} \in G_{k}^{\prime}\left(\mathbb{C}^{n}\right)(\nu \in \mathbb{N})$,

$$
L_{\nu} \stackrel{\tau}{\rightarrow} L \Leftrightarrow L_{\nu}=z_{0}^{\nu}+\sum_{j=1}^{k} \mathbb{C} z_{j}^{\nu} \text { for some } z_{j}^{\nu} \rightarrow z_{j}(j=0,1, \ldots, k) .
$$

On the other hand, since $G_{k}^{\prime}\left(\mathbb{C}^{n}\right) \subset \mathcal{F}_{\mathbb{C}^{n}}$, we have the subspace topology in $G_{k}^{\prime}\left(\mathbb{C}^{n}\right)$ (see also [12]). The following lemma states that these two topologies coincide.

Lemma 1.2. Suppose that $\left(L_{\nu}\right)$ is a sequence in $G_{k}^{\prime}\left(\mathbb{C}^{n}\right)$ and let $L \in$ $G_{k}^{\prime}\left(\mathbb{C}^{n}\right)$. Then

$$
L_{\nu} \stackrel{\tau}{\rightarrow} L \Leftrightarrow L_{\nu} \stackrel{\mathcal{F}_{\mathbb{C}^{n}}}{\longrightarrow} L .
$$

Proof. Assume that $L_{\nu} \stackrel{\tau}{\rightarrow} L$, where $L_{\nu}, L \in G_{k}^{\prime}\left(\mathbb{C}^{n}\right)$. Let $L=z_{0}+$ $\sum_{j=1}^{k} \mathbb{C} z_{j}$. We know that $L_{\nu}=z_{0}^{\nu}+\sum_{j=1}^{k} \mathbb{C} z_{j}^{\nu}$ for some $z_{j}^{\nu} \rightarrow z_{j}(j=$ $0,1, \ldots, k)$. Obviously, for any $x \in L$ there exist $x^{\nu} \in L_{\nu}$ such that $x^{\nu} \rightarrow x$. Take now a compact set $K \subset \mathbb{C}^{n}$ such that $L \cap K=\emptyset$. We need to show that for some $\nu_{0} \in \mathbb{N}$ we have $L_{\nu} \cap K=\emptyset$ whenever $\nu \geq \nu_{0}$. Suppose that this is not the case. Passing to a subsequence if necessary we may assume that $L_{\nu} \cap K \neq \emptyset$ for any $\nu$. So let $y^{\nu} \in L_{\nu} \cap K$. We have

$$
y^{\nu}=z_{0}^{\nu}+\sum_{j=1}^{k} \alpha_{j}^{\nu} z_{j}^{\nu}
$$

for some $\alpha_{j}^{\nu} \in \mathbb{C}(j=1, \ldots, k)$. Take $1 \leq l_{1}<\cdots<l_{k} \leq n$ such that $\left.\operatorname{det}\left[\pi\left(z_{1}\right), \ldots, \pi\left(z_{k}\right)\right)\right] \neq 0$, where

$$
\pi: \mathbb{C}^{n} \ni\left(t_{1}, \ldots, t_{n}\right) \mapsto\left(t_{l_{1}}, \ldots, t_{l_{k}}\right) \in \mathbb{C}^{k} .
$$

Then for each $\nu$ large enough we have $\left.\mid \operatorname{det}\left[\pi\left(z_{1}^{\nu}\right), \ldots, \pi\left(z_{k}^{\nu}\right)\right)\right] \mid \geq M$, where $M$ is a positive constant. We can treat $(\star)$ as a system of linear equations, where $\alpha_{j}^{\nu}$ are the unknowns. By Cramer's rule applied to the equation $\pi\left(y^{\nu}\right)=$ $\pi\left(z_{0}^{\nu}\right)+\sum_{j=1}^{k} \alpha_{j}^{\nu} \pi\left(z_{j}^{\nu}\right)$, all $\alpha_{j}^{\nu}$ are bounded. Passing again to a subsequence if necessary we can assume that $\alpha_{j}^{\nu} \rightarrow \alpha_{j} \in \mathbb{C}$. This is a contradiction, since $y:=z_{0}+\sum_{j=1}^{k} \alpha_{j} z_{j} \in L \cap K$. 
Assume now that $L_{\nu} \stackrel{\mathcal{F}_{\mathbb{C} n}}{\longrightarrow} L$, where $L_{\nu}, L \in G_{k}^{\prime}\left(\mathbb{C}^{n}\right)$ and $L=z_{0}+$ $\sum_{j=1}^{k} \mathbb{C} z_{j}$. Take $z_{0}^{\nu} \in L_{\nu}$ such that $z_{0}^{\nu} \rightarrow z_{0}$, and $a_{j}^{\nu} \in L_{\nu}$ such that $a_{j}^{\nu} \rightarrow$ $z_{0}+z_{j}$ for $j=1, \ldots, k$. Put $z_{j}^{\nu}:=a_{j}^{\nu}-z_{0}^{\nu}(j=1, \ldots, k)$. Obviously, $z_{j}^{\nu} \rightarrow z_{j}$. Moreover, $z_{1}^{\nu}, \ldots, z_{k}^{\nu} \in L_{\nu}-z_{0}^{\nu}$ are linearly independent for all $\nu$ large enough and thus $L_{\nu}=z_{0}^{\nu}+\sum_{j=1}^{k} \mathbb{C} z_{j}^{\nu}$.

Let $\Omega \subset \mathbb{C}^{n}$ be an open set. We denote by $\mathcal{A}_{k}(\Omega)$ the set of all analytic sets in $\Omega$ of pure dimension $k\left({ }^{1}\right)$. Since $\mathcal{A}_{k}(\Omega) \subset \mathcal{F}_{\Omega}$, we have the subspace topology in $\mathcal{A}_{k}(\Omega)$. Recall the following theorem due to Tworzewski and Winiarski.

ThEOREM 1.3 (Tworzewski, Winiarski). Let $W \in \mathcal{A}_{k}(\Omega)$ and $V_{0} \in$ $\mathcal{A}_{n-k}(\Omega)$ be such that $V_{0} \cap W \in \mathcal{A}_{0}(\Omega)$. Then the mapping

$$
\mathcal{A}_{n-k}(\Omega) \ni V \mapsto V \cap W \in \mathcal{F}_{W}
$$

is continuous at $V_{0}$.

Proof. Cf. [10, Corollary 2].

Corollary 1.4. Let $L \in G_{n-k}^{\prime}\left(\mathbb{C}^{n}\right)$ and $A \in \mathcal{A}_{k}(U)$, where $U \subset \mathbb{C}^{n}$ is open. Suppose that $L \cap A$ contains (at least) $p$ isolated points. Then

- There exists an open neighbourhood of $L$ in $G_{n-k}^{\prime}\left(\mathbb{C}^{n}\right)$ such that for any $H$ from this neighbourhood we have

$$
\#(H \cap A) \geq p .
$$

- There exists an open neighbourhood of $A$ in $\mathcal{A}_{k}(U)$ such that for any $V$ from this neighbourhood we have

$$
\#(L \cap V) \geq p .
$$

Proof. We prove only the first part, since the proof of the second is quite similar. Let $\Omega \subset U$ be open, bounded and such that $\#(L \cap \Omega \cap A)=p$. Suppose that there exist $H_{\nu} \in G_{n-k}^{\prime}\left(\mathbb{C}^{n}\right)$ such that $H_{\nu} \rightarrow L$ and $\#\left(H_{\nu} \cap A\right)$ $<p$. Obviously, $H_{\nu} \cap \Omega \rightarrow L \cap \Omega$ in $\mathcal{F}_{\Omega}$. By the previous theorem, we get $H_{\nu} \cap \Omega \cap A \rightarrow L \cap \Omega \cap A$ in $\mathcal{F}_{\Omega \cap A}$, but this is impossible.

2. Degree of analytic set. Let $V \subset \mathbb{C}^{n}$ be an algebraic set of pure dimension $k$. Recall that we define the degree $\operatorname{deg} V$ of $V$ as the unique number $p$ such that $\#(L \cap V)=p$ for each $L$ from some open dense $\left(^{2}\right)$ subset of $G_{n-k}^{\prime}\left(\mathbb{C}^{n}\right)$ (cf. [6]).

REMARK 2.1. Corollary 1.4 implies that

$$
\operatorname{deg} V=\sup \left\{\#(L \cap V): L \in G_{n-k}^{\prime}\left(\mathbb{C}^{n}\right), \#(L \cap V)<\infty\right\} .
$$

$\left({ }^{1}\right)$ We assume that $\emptyset$ is of pure dimension $k$ for any $k \in \mathbb{N}$.

$\left({ }^{2}\right)$ In the topology $\tau$ which is equivalent by Lemma 1.2 to the topology induced from $\mathcal{F}_{\mathbb{C}^{n}}$. 
Recall the following

THEOREM 2.2. If $A$ is an analytic set of dimension $k$ in some open set $\Omega \subset \mathbb{C}^{n}$, then $L \cap A$ is discrete for each $L$ from a dense subset of $G_{n-k}\left(\mathbb{C}^{n}\right)\left({ }^{3}\right)$.

Proof. Cf. [6, p. 185].

Assume now that $\Omega \subset \mathbb{C}^{n}$ is an open set and let $A$ be an analytic subset of $\Omega$. We define $\operatorname{dg}(A ; \Omega)$ as follows:

(1) If $A$ is of pure dimension $k$, then we put

$\operatorname{dg}(A ; \Omega):=\sup \left\{\#(L \cap A): L \in G_{n-k}^{\prime}\left(\mathbb{C}^{n}\right), L \cap A\right.$ is a discrete set $\}\left(^{4}\right)$.

(2) If $A=\bigcup A_{l}$, where $A_{l}$ is the union of all irreducible components of $A$ of dimension $l$, then we put

$$
\operatorname{dg}(A ; \Omega):=\sum_{l} \operatorname{dg}\left(A_{l} ; \Omega\right) \in \mathbb{N} \cup\{\infty\} .
$$

REMARK 2.3. We have the following properties:

- If $A$ is an analytic subset of $\Omega$, then

$$
\operatorname{dg}(A ; \Omega) \leq \sum_{i} \operatorname{dg}\left(C_{i} ; \Omega\right),
$$

where $A=\bigcup C_{i}$ is the decomposition of $A$ into irreducible components.

- If $V \subset \mathbb{C}^{n}$ is algebraic of pure dimension, then

$$
\operatorname{dg}\left(V ; \mathbb{C}^{n}\right)=\operatorname{deg} V .
$$

Proof. The first part is trivial. The second follows immediately from Remark 2.1.

Let us add that for any analytic set $A \subset \mathbb{C}^{n}$ we have:

$$
A \text { is algebraic } \Leftrightarrow \operatorname{dg}\left(A ; \mathbb{C}^{n}\right)<\infty \text {. }
$$

This is a consequence of the following two theorems. The first one is due to Gruman. The second is a more precise version of Theorem 2.2.

Theorem 2.4 (Gruman). Suppose that $A \in \mathcal{A}_{k}\left(\mathbb{C}^{n}\right)$. If $L \cap A$ is finite for any $L \in E$, where $E \subset G_{n-k}\left(\mathbb{C}^{n}\right)$ is a set of positive volume, then $A$ is algebraic.

Proof. Cf. [7, Corollary 4.4].

$\left({ }^{3}\right) G_{n-k}\left(\mathbb{C}^{n}\right)$ denotes the Grassmann manifold of all $(n-k)$-dimensional subspaces of $\mathbb{C}^{n}$.

$\left({ }^{4}\right)$ Note that Theorem 2.2 implies in particular that the family of all $L \in G_{n-k}^{\prime}\left(\mathbb{C}^{n}\right)$ such that $L \cap A$ is a discrete set is nonempty. 
THeOREM 2.5. Assume that $A$ is an analytic set of dimension $k$ in some open set $\Omega \subset \mathbb{C}^{n}$. Then there exists a subset $S \subset G_{n-k}\left(\mathbb{C}^{n}\right)$ of measure zero such that $L \cap A$ is discrete for each $L \in G_{n-k}\left(\mathbb{C}^{n}\right) \backslash S$.

Proof. This follows from a more general result stated in the Appendix.

Let $N$ be a Nash subset of an open set $\Omega \subset \mathbb{C}^{n}$ (see [9] for the definition and properties of Nash sets). Recall a characterization of Nash sets:

Theorem 2.6 (Tworzewski). If $N \neq \emptyset$ is irreducible, then there exists an irreducible algebraic set $V \subset \mathbb{C}^{n}$ such that $N$ is an irreducible component of $V \cap \Omega$.

Proof. Cf. [9, Theorem 2.10].

REMARK 2.7. It is easy to see that the algebraic set $V$ in the above theorem is unique.

We define $\operatorname{deg}(N ; \Omega)$ as follows:

(1) If $N \neq \emptyset$ is irreducible, then we put

$$
\operatorname{deg}(N ; \Omega):=\operatorname{deg} V,
$$

where $V$ is from Theorem 2.6. Moreover, we put $\operatorname{deg}(\emptyset ; \Omega):=0$.

(2) If $N=\bigcup N_{i}$ is the decomposition into irreducible components $\left(^{5}\right)$, then we put

$$
\operatorname{deg}(N ; \Omega):=\sum_{i} \operatorname{deg}\left(N_{i} ; \Omega\right) \in \mathbb{N} \cup\{\infty\}
$$

REMARK 2.8. We have the following properties:

(1) If $V \subset \mathbb{C}^{n}$ is algebraic of pure dimension, then

$$
\operatorname{deg}\left(V ; \mathbb{C}^{n}\right)=\operatorname{deg} V
$$

(2) If $N$ is a Nash subset of $\Omega \subset \mathbb{C}^{n}$, then

$$
\operatorname{dg}(N ; \Omega) \leq \operatorname{deg}(N ; \Omega) .
$$

The above inequality may be strict even if $N$ is irreducible.

Proof. Part (1) follows from the following fact: if $V=V_{1} \cup \cdots \cup V_{p}$ is the decomposition into irreducible components, then $\operatorname{deg} V=\operatorname{deg} V_{1}+\cdots+$ $\operatorname{deg} V_{p}$ (cf. [6, p. 310]).

Let us pass to (2). By Remark 2.3, we may restrict ourselves to the case when $N \neq \emptyset$ is irreducible. Put $k:=\operatorname{dim} N$. Take $L \in G_{n-k}^{\prime}\left(\mathbb{C}^{n}\right)$ such that $L \cap N$ is a discrete set. We need to show that $\#(L \cap N) \leq \operatorname{deg}(N ; \Omega)=\operatorname{deg} V$, where $V$ is the unique algebraic set as in Theorem 2.6. Suppose that this is not the case. Corollary 1.4 implies that $\#(H \cap N)>\operatorname{deg} V$ for each $H$ in

$\left({ }^{5}\right)$ They are Nash subsets of $\Omega$ (cf. [9, Theorem 2.11]). 
some nonempty open set in $G_{n-k}^{\prime}\left(\mathbb{C}^{n}\right)$. Hence $\#(H \cap V)>\operatorname{deg} V$. This gives a contradiction.

To see that the inequality in (2) may be strict consider the set $A:=$ $\left\{(z, y) \in \mathbb{C}^{2}: y=z^{2}+z^{3}\right\}$. Note that $\operatorname{deg}(A \cap \Omega ; \Omega) \geq 3$ whenever $\Omega \subset \mathbb{C}^{2}$ is an open set such that $A \cap \Omega \neq \emptyset$. Put $\Omega:=\{|z|<1 / 3\} \times \mathbb{C}$. Suppose that $\operatorname{dg}(A \cap \Omega ; \Omega) \geq 3$. Since $A \cap \Omega$ is of pure dimension 1 , it follows that there exist some $a, b \in \mathbb{C}$ such that the equation $a z+b=z^{2}+z^{3}$ has three different roots $z_{1}, z_{2}, z_{3}$ in $\{|z|<1 / 3\}$. We obtain a contradiction, because $z_{1}+z_{2}+z_{3}=-1$.

3. Limits of analytic sets. Recall the main result of [11].

Theorem 3.1 (Tworzewski, Winiarski). The set $\mathcal{H}_{d}^{k}\left(\mathbb{C}^{n}\right):=\left\{V \in \mathcal{A}_{k}\left(\mathbb{C}^{n}\right)\right.$ : $V$ is algebraic, $\operatorname{deg} V \leq d\}$ is compact in $\mathcal{F}_{\mathbb{C}^{n}}$.

Proof. Cf. [11, Theorem 2].

Tworzewski and Winiarski use among others the following tools:

Theorem 3.2 (Bishop). Let $\Omega \subset \mathbb{C}^{n}$ be open and let $A_{\nu} \in \mathcal{A}_{k}(\Omega)$, $\nu=1,2, \ldots$. If the $2 k$-dimensional volumes of $A_{\nu}$ are finite and bounded by a positive constant independent of $\nu$ and $A_{\nu} \rightarrow A$ in $\mathcal{F}_{\Omega}$, then $A \in \mathcal{A}_{k}(\Omega)$.

Proof. Cf. [2, Theorem 1]. See also [8, Theorem C].

Theorem 3.3 (Griffiths). Let $V \subset \mathbb{C}^{n}$ be an algebraic set of pure dimension $k$. Then

$$
\operatorname{vol}_{2 k}(V \cap B[r]) \leq \alpha(k) \cdot \operatorname{deg} V \cdot r^{2 k}
$$

for any $r>0$, where $B[r]:=\left\{z \in \mathbb{C}^{n}:\|z\|<r\right\}$ and $\alpha(k)$ is a positive constant independent of $V$.

Proof. Cf. [4, Theorems 1.3 and 1.8].

We will now prove a refined version of Theorem 3.1.

Theorem 3.4. Suppose that $A \subset \mathbb{C}^{n}$ and let $\Omega$ be open in $\mathbb{C}^{n}$. Assume that there exists a sequence $\left(N_{\nu}\right)$ of Nash subsets of $\Omega$ such that $N_{\nu} \rightarrow A \cap \Omega$ in $\mathcal{F}_{\Omega}$ and $\operatorname{deg}\left(N_{\nu} ; \Omega\right) \leq d$. Then $A \cap \Omega$ is a Nash subset of $\Omega$. Moreover, if additionally $\Omega=\mathbb{C}^{n}$ or $A$ is analytic irreducible such that $A \cap \Omega \neq \emptyset$, then $A$ is algebraic.

Proof. Since $\operatorname{deg}\left(N_{\nu} ; \Omega\right) \leq d$, each $N_{\nu}$ contains at most $d$ irreducible components. Passing to a subsequence if necessary we may assume that for each $\nu$ we have the following decomposition into irreducible components:

$$
N_{\nu}=\bigcup_{i=1}^{s} \bigcup_{j=1}^{p_{i}} N_{\nu}^{i, j},
$$


where for any $i \in\{1, \ldots, s\}, N_{\nu}^{i, j}\left(j=1, \ldots, p_{i}\right)$ denote the components of dimension $k_{i}$. Obviously,

$$
\sum_{i=1}^{s} \sum_{j=1}^{p_{i}} \operatorname{deg}\left(N_{\nu}^{i, j} ; \Omega\right) \leq d .
$$

For each $N_{\nu}^{i, j}$ take the unique algebraic set $V_{\nu}^{i, j}$ as in Theorem 2.6. Then:

- $N_{\nu}^{i, j}$ is an irreducible component of $V_{\nu}^{i, j} \cap \Omega$,

- $\operatorname{deg} V_{\nu}^{i, j}=\operatorname{deg}\left(N_{\nu}^{i, j} ; \Omega\right) \leq d$,

- $V_{\nu}^{i, j}$ is irreducible of dimension $k_{i}$.

Again passing to a subsequence if necessary we may assume that $N_{\nu}^{i, j} \rightarrow N^{i, j}$ in $\mathcal{F}_{\Omega}$ and $V_{\nu}^{i, j} \rightarrow V^{i, j}$ in $\mathcal{F}_{\mathbb{C}^{n}}$. It follows from Theorem 3.1 that $V^{i, j}$ is an algebraic set of pure dimension $k_{i}$. By Theorem 3.3, we get

$$
\operatorname{vol}_{2 k_{i}}\left(V_{\nu}^{i, j} \cap B[r]\right) \leq \alpha\left(k_{i}\right) \cdot d \cdot r^{2 k_{i}}
$$

and thus

$$
\operatorname{vol}_{2 k_{i}}\left(N_{\nu}^{i, j} \cap B[r]\right) \leq \alpha\left(k_{i}\right) \cdot d \cdot r^{2 k_{i}} .
$$

Theorem 3.2 implies that $N^{i, j} \in \mathcal{A}_{k_{i}}(\Omega)$. Since $N^{i, j} \subset V^{i, j} \cap \Omega$ and $V^{i, j}$ is of pure dimension $k_{i}, N^{i, j}$ is a union of some irreducible components of $V^{i, j} \cap \Omega$. Therefore $N^{i, j}$ is a locally finite (in $\Omega$ ) union of Nash sets. By Proposition 2.6 in [9], $N^{i, j}$ is a Nash subset of $\Omega$. Now it is enough to see that

$$
A \cap \Omega=\bigcup_{i=1}^{s} \bigcup_{j=1}^{p_{i}} N^{i, j} .
$$

It follows from the proof that for $\Omega=\mathbb{C}^{n}, A$ is algebraic. If $A$ is analytic irreducible and $A \cap \Omega \neq \emptyset$, then $A$ is algebraic as well (cf. [9, Theorems 2.9 and 2.12]).

Corollary 3.5. Let $\Omega \subset \mathbb{C}^{n}$ be open and let $N \subset \Omega \times \mathbb{C}^{m}$ be a Nash set with a finite number of irreducible components. Then $\overline{\pi(N)} \Omega$ is Nash in $\Omega$, where $\pi: \mathbb{C}^{n} \times \mathbb{C}^{m} \rightarrow \mathbb{C}^{n}$ denotes the natural projection.

Proof. It is enough to repeat the proof of Corollary in [11].

REMARK 3.6. One cannot omit the assumption that $N$ has a finite number of irreducible components. To see this consider $N:=\{(1 / \nu, \nu): \nu \in \mathbb{N}\}$ $(n=m=1)$. In this case we have $\overline{\pi(N)}=\{1 / \nu: \nu \in \mathbb{N}\} \cup\{0\}$, which is not even analytic.

If we replace $\operatorname{deg}\left(N_{\nu} ; \Omega\right)$ in Theorem 3.4 by $\operatorname{dg}\left(N_{\nu} ; \Omega\right)$, then the conclusion is no longer true, even if we assume that $N_{\nu}$ are irreducible and $A$ is analytic irreducible. We give an example in the next section. However, we can prove the following theorem. 
Theorem 3.7. Let $A \in \mathcal{A}_{k}\left(\mathbb{C}^{n}\right)$ and let $U_{1} \subset U_{2} \subset \cdots$ be a sequence of open subsets of $\mathbb{C}^{n}$ such that $\bigcup U_{i}=\mathbb{C}^{n}$. Assume that there is some positive integer $d$ such that for each $i$ there exists a sequence $\left(A_{i j}\right)$ in $\mathcal{A}_{k}\left(U_{i}\right)$ with the following properties:

- $A_{i j} \rightarrow A \cap U_{i}$ in $\mathcal{F}_{U_{i}}$,

- $\operatorname{dg}\left(A_{i j} ; U_{i}\right) \leq d$.

Then $A$ is algebraic.

Proof. Fix $i \in \mathbb{N}$. By Theorem 2.5, we get a subset $S_{i} \subset G_{n-k}\left(\mathbb{C}^{n}\right)$ of measure zero such that the sets $L \cap A_{i j}(j=1,2, \ldots)$ and $L \cap A \cap U_{i}$ are discrete for each $L \in G_{n-k}\left(\mathbb{C}^{n}\right) \backslash S_{i}$. Fix $L \in G_{n-k}\left(\mathbb{C}^{n}\right) \backslash S_{i}$. Since $\operatorname{dg}\left(A_{i j} ; U_{i}\right) \leq d$, we have $\#\left(L \cap A_{i j}\right) \leq d$. Assume that $\#\left(L \cap A \cap U_{i}\right)>d$. Then $L \cap A \cap U_{i}$ contains $d+1$ isolated points. Now it is enough to use Corollary 1.4 to get a contradiction. To summarize, for each $i \in \mathbb{N}$ and $L \in G_{n-k}\left(\mathbb{C}^{n}\right) \backslash S_{i}$ we have

$$
\#\left(L \cap A \cap U_{i}\right) \leq d .
$$

Put $S:=\bigcup S_{i}$. For any $L \in G_{n-k}\left(\mathbb{C}^{n}\right) \backslash S$ we have $\#(L \cap A) \leq d$. Now the result follows from Theorem 2.4.

4. An example. We will now give an example announced in the previous section. First, we need some lemmata.

For any $\nu \in \mathbb{N}$ put

$$
h_{\nu}(z):=\frac{z^{\nu}}{\nu !}+\frac{z^{\nu-1}}{(\nu-1) !}+\cdots+z+1 .
$$

Lemma 4.1. If the equation $h_{\nu}(z)=a z+b$, where $a, b \in \mathbb{C}$, has two different roots in $\{|z| \leq 1\}$, then $|a| \leq e$ and $|b| \leq 2 e$. The same is true for the equation $e^{z}=a z+b$.

Proof. Let $u, w \in\{|z| \leq 1\}$ be two different roots of the equation $h_{\nu}(z)=$ $a z+b$. Then

$$
a(u-w)=\sum_{j=1}^{\nu} \frac{1}{j !}\left(u^{j}-w^{j}\right) .
$$

Therefore we have

and

$$
|a| \leq 1+\frac{1}{2 !} \cdot 2+\frac{1}{3 !} \cdot 3+\cdots+\frac{1}{\nu !} \cdot \nu<e
$$

$$
|b| \leq\left|h_{\nu}(u)\right|+|a u| \leq 2 e .
$$

Just in the same way we prove the second statement.

Lemma 4.2. There exists a positive integer $d$ such that for any $a, b \in \mathbb{C}$,

$$
\#\left\{z \in \mathbb{C}:|z| \leq 1, e^{z}=a z+b\right\} \leq d .
$$


Proof. By Lemma 4.1, we may assume that $|a| \leq e$ and $|b| \leq 2 e$. Consider the set

$$
K:=\left\{u \in \mathbb{R}^{6}: e^{x_{1}+i y_{1}}=\left(x_{2}+i y_{2}\right)\left(x_{1}+i y_{1}\right)+x_{3}+i y_{3}\right\} \cap T,
$$

where $T:=\left\{\left\|u_{1}\right\| \leq 1,\left\|u_{2}\right\| \leq e,\left\|u_{3}\right\| \leq 2 e\right\} \subset \mathbb{R}^{6}, u=\left(u_{1}, u_{2}, u_{3}\right)$ and $u_{j}=\left(x_{j}, y_{j}\right) \in \mathbb{R}^{2}$ for $j=1,2,3$. Note that $K$ is a semianalytic $\left(^{6}\right)$ compact subset of $\mathbb{R}^{6}$. Since for any $\left(u_{2}, u_{3}\right) \in \mathbb{R}^{2} \times \mathbb{R}^{2}$ the fibre $K_{\left(u_{2}, u_{3}\right)}$ is finite, there exists a positive integer $d$ such that $\# K_{\left(u_{2}, u_{3}\right)} \leq d$.

LEMma 4.3. There exists $\nu_{0} \in \mathbb{N}$ such that for any $\nu \geq \nu_{0}$ and any $a, b \in \mathbb{C}$ the equation $h_{\nu}(z)=a z+b$ has at most $2 d$ distinct roots in the set $\{|z| \leq 1\}$, where $d$ is from the previous lemma.

Proof. Suppose that this is not the case. Then we can find a subsequence $\nu_{k} \rightarrow \infty$ and sequences $a_{\nu_{k}}, b_{\nu_{k}}$ of complex numbers such that for any $k$ the equation $h_{\nu_{k}}(z)=a_{\nu_{k}} z+b_{\nu_{k}}$ has at least $2 d+1$ distinct roots in the set $\{|z| \leq 1\}$. Denote these roots by $y_{1}^{\nu_{k}}, \ldots, y_{2 d+1}^{\nu_{k}}$. Lemma 4.1 implies that $a_{\nu_{k}}, b_{\nu_{k}}$ are bounded. Passing to a subsequence if necessary we may assume that

$$
y_{j}^{\nu_{k}} \rightarrow y_{j}(j=1, \ldots, 2 d+1), \quad a_{\nu_{k}} \rightarrow a, \quad b_{\nu_{k}} \rightarrow b,
$$

for some $y_{j} \in\{|z| \leq 1\}$ and $a, b \in \mathbb{C}$. Put $g(z)=e^{z}-a z-b$ and $g_{\nu_{k}}(z)=$ $h_{\nu_{k}}(z)-a_{\nu_{k}} z-b_{\nu_{k}}$ for $z \in \mathbb{C}$. Obviously, $g_{\nu_{k}} \rightarrow g$ uniformly in $\{|z| \leq 2\}$. It is easy to see that $g\left(y_{j}\right)=0$ for $j=1, \ldots, 2 d+1$. By the previous lemma, at least three numbers among $y_{1}, \ldots, y_{2 d+1}$ are equal, say $y_{1}=y_{2}=y_{3}$. By the Hurwitz theorem, $y_{1}$ is a zero of $g$ of multiplicity at least 3 . This is impossible, because $g^{\prime \prime}\left(y_{1}\right)=e^{y_{1}} \neq 0$.

EXAmple 4.4. Let $\Omega:=\{|z|<1\} \times \mathbb{C} \subset \mathbb{C}^{2}$. For any $\nu \in \mathbb{N}$ put

$$
N_{\nu}:=\left\{(z, y) \in \mathbb{C}^{2}: y=h_{\nu}(z)\right\} \cap \Omega
$$

and

$$
A:=\left\{(z, y) \in \mathbb{C}^{2}: y=e^{z}\right\} .
$$

Note that each $N_{\nu}$ is an irreducible Nash subset of $\Omega$ and $A$ is an irreducible analytic set in $\mathbb{C}^{2}$. Moreover, $\operatorname{dg}\left(N_{\nu} ; \Omega\right) \leq 2 d$ for $\nu \geq \nu_{0}$ (cf. Lemma 4.3) and $N_{\nu} \rightarrow A \cap \Omega$ in $\mathcal{F}_{\Omega}$. Nevertheless, $A$ is not algebraic.

5. Appendix. In this section we present a general result concerning o-minimal structures $\left({ }^{7}\right)$ from which Theorem 2.5 follows immediately.

$\left({ }^{6}\right)$ See [5] and [1] for the definition and properties of semianalytic sets.

$\left({ }^{7}\right)$ The definition and properties of o-minimal structures can be found in [3]. 
Let $J$ denote the set of all increasing functions $\alpha:\{1, \ldots, n-k\} \rightarrow$ $\{1, \ldots, n\}$. For each $\alpha \in J$ put

$$
V_{\alpha}:=\sum_{j=1}^{n-k} \mathbb{C} e_{\alpha(j)}, \quad W_{\alpha}:=\sum_{\nu \notin i m \alpha} \mathbb{C} e_{\nu},
$$

where $e_{1}, \ldots, e_{n}$ is the usual basis in $\mathbb{C}^{n}$. We denote by $\Omega\left(V_{\alpha}\right)$ the subset of $G_{k}\left(\mathbb{C}^{n}\right)$ consisting of all linear complements of $V_{\alpha}$. Recall that the family of maps

$$
\varphi_{\alpha}: \mathcal{L}\left(W_{\alpha}, V_{\alpha}\right) \ni L \mapsto \widehat{L} \in \Omega\left(V_{\alpha}\right),
$$

where $\alpha \in J$ and $\widehat{L}:=\left\{y+L(y): y \in W_{\alpha}\right\}$, is an inverse atlas on $G_{k}\left(\mathbb{C}^{n}\right)\left(^{8}\right)$.

We will use the following result:

TheOrem 5.1. Let $f: X \rightarrow \mathbb{R}^{m}$ be definable in some o-minimal structure, where $X \subset \mathbb{R}^{p}$. For each $j \in\{0,1, \ldots, p\}$ put $A_{j}:=\left\{x \in \mathbb{R}^{m}\right.$ : $\left.\operatorname{dim} f^{-1}(x)=j\right\}$. Then

$$
\operatorname{dim} f^{-1}\left(A_{j}\right)=\operatorname{dim} A_{j}+j .
$$

Proof. Cf. [3, p. 66].

TheOREM 5.2. Suppose that $B \subset \mathbb{C}^{n}$ is definable (as a subset of $\mathbb{R}^{2 n}$ ) in some o-minimal structure and $\operatorname{dim} B \leq 2(n-k)$. Then there exists a positive integer $d$ and a set $S \subset G_{k}\left(\mathbb{C}^{n}\right)$ of measure zero such that $\#(H \cap B) \leq d$ for any $H \in G_{k}\left(\mathbb{C}^{n}\right) \backslash S$.

Proof. It is enough to show that for any $\alpha \in J$ we can find a positive integer $d_{\alpha}$ and a subset $S_{\alpha} \subset \mathcal{L}\left(W_{\alpha}, V_{\alpha}\right)$ of measure zero such that $\#(\widehat{L} \cap B) \leq d_{\alpha}$ whenever $L \in \mathcal{L}\left(W_{\alpha}, V_{\alpha}\right) \backslash S_{\alpha}$. We will prove this for $V_{\alpha}=\{0\} \times \mathbb{C}^{n-k} \subset \mathbb{C}^{n}$ (the general case is analogous). Put $V:=V_{\alpha}, W:=W_{\alpha}=\mathbb{C}^{k} \times\{0\} \subset \mathbb{C}^{n}$. Define

$$
F: \mathbb{C}^{k} \times \mathcal{L}(W, V) \ni(u, L) \mapsto(u, 0)+L(u, 0) \in \mathbb{C}^{n} .
$$

Since $\mathbb{C}^{k}=\mathbb{R}^{2 k}$ and $\mathcal{L}(W, V)$ can be identified with $\mathbb{R}^{2 k(n-k)}, F^{-1}(B)$ may be regarded as a subset of $\mathbb{R}^{2 k} \times \mathbb{R}^{2 k(n-k)}$. Obviously, $F^{-1}(B)$ is then definable, for $F$ and $B$ are. For fixed $u \in \mathbb{C}^{k}$ we have the linear mapping

$$
h_{u}: \mathcal{L}(W, V) \ni L \mapsto L(u, 0) \in V .
$$

Note the following facts:

(1) If $u \neq 0$, then for any $v \in V$ the set $h_{u}^{-1}(v)$ is a $(k-1)(n-k)$ dimensional affine complex subspace.

(2) $h_{0}^{-1}(0)=\mathcal{L}(W, V)$ and $h_{0}^{-1}(v)=\emptyset$ if $v \neq 0$.

$\left(^{8}\right) \mathcal{L}(X, Y)$ denotes the space of all linear maps $X \rightarrow Y$. 
It is easy to see that for any $x=\left(x^{\prime}, x^{\prime \prime}\right) \in \mathbb{C}^{k} \times \mathbb{C}^{n-k}=\mathbb{C}^{n}$ we have $F^{-1}(x)=\left\{x^{\prime}\right\} \times h_{x^{\prime}}^{-1}\left(0, x^{\prime \prime}\right)$. Therefore

$$
\operatorname{dim} F^{-1}(x) \leq 2(k-1)(n-k) \quad \text { if } x \neq 0, \quad \operatorname{dim} F^{-1}(0)=2 k(n-k) .\left({ }^{9}\right)
$$

By Theorem 5.1, we get $\operatorname{dim} F^{-1}(B \backslash\{0\}) \leq 2 k(n-k)$. Combining this with the fact that $\operatorname{dim} F^{-1}(0)=2 k(n-k)$ we have

$$
\operatorname{dim} F^{-1}(B) \leq 2 k(n-k)
$$

Hence there exists a positive integer $s$ and a nowhere dense and definable $\left({ }^{10}\right)$ set $Z \subset \mathcal{L}(W, V)$ such that $\# F^{-1}(B)_{L} \leq s$ for any $L \in \mathcal{L}(W, V) \backslash Z$, where $F^{-1}(B)_{L}$ is the fibre of $F^{-1}(B)$ over $L$. Consider the bijection

$$
g_{L}: \mathbb{C}^{k} \ni u \mapsto(u, 0)+L(u, 0) \in \widehat{L} .
$$

Since $F^{-1}(B)_{L}=g_{L}^{-1}(\widehat{L} \cap B)$, we obtain

$$
\# F^{-1}(B)_{L}=\#(\widehat{L} \cap B) \text {. }
$$

To summarize, for any $L \in \mathcal{L}(W, V) \backslash Z$ we have $\#(\widehat{L} \cap B) \leq s$.

TheOREM 5.3. Let $X \subset \mathbb{C}^{n}$. Suppose that for each $x \in X$ there exists an open neighbourhood $U_{x} \subset \mathbb{C}^{n}$ of $x$ such that $U_{x} \cap X$ is definable in some o-minimal structure (which may depend on $x)$ and $\operatorname{dim}\left(U_{x} \cap X\right) \leq 2(n-k)$. Then there exists a subset $S \subset G_{k}\left(\mathbb{C}^{n}\right)$ of measure zero such that $H \cap X$ is a discrete set for any $H \in G_{k}\left(\mathbb{C}^{n}\right) \backslash S$.

Proof. It is enough to use the previous theorem and the following facts:

- $X$ is a second-countable space,

- a countable union of sets of measure zero is of measure zero.

REMARK 5.4. Theorem 2.5 follows immediately from Theorem 5.3.

Acknowledgements. We would like to thank Professor Wiesław Pawłucki for helpful discussions and comments about the paper.

\section{References}

[1] E. Bierstone and P. Milman, Semianalytic and subanalytic sets, Inst. Hautes Études Sci. Publ. Math. 67 (1988), 5-42.

[2] E. Bishop, Conditions for the analyticity of certain sets, Michigan Math. J. 11 (1964), 289-304.

[3] L. van den Dries, Tame Topology and o-Minimal Structures, London Math. Soc. Lecture Note Ser. 248, Cambridge Univ. Press, 1998.

[4] P. A. Griffiths, Entire Holomorphic Mappings in One and Several Complex Variables, Princeton Univ. Press, 1976.

$\left({ }^{9}\right)$ Here dim denotes the dimension of a definable set.

$\left({ }^{10}\right)$ And thus of measure zero. 
[5] S. Łojasiewicz, Ensembles semi-analytiques, Lecture Notes, IHES, Bures-sur-Yvette, France, 1965; http://perso.univ-rennes1.fr/michel.coste/Lojasiewicz.pdf.

[6] -, Wstęp do geometrii analitycznej zespolonej, Biblioteka Mat. 68, PWN, Warszawa, 1988; English transl.: Introduction to Complex Analytic Geometry, Birkhäuser, 1991.

[7] R. E. Molzon, B. Shiffman and N. Sibony, Average growth estimates for hyperplane sections of entire analytic sets, Math. Ann. 257 (1981), 43-59.

[8] G. Stolzenberg, Volumes, Limits and Extensions of Analytic Varieties, Lecture Notes in Math. 19, Springer, 1966.

[9] P. Tworzewski, Intersections of analytic sets with linear subspaces, Ann. Scuola Norm. Sup. Pisa Cl. Sci (4) 17 (1990), 227-271.

[10] P. Tworzewski and T. Winiarski, Continuity of intersection of analytic sets, Ann. Polon. Math. 42 (1983), 387-393.

[11] - - - Limits of algebraic sets of bounded degree, Univ. Iagell. Acta Math. 24 (1984), 151-153.

[12] T. Winiarski, Continuity of total number of intersection, Ann. Polon. Math. 47 (1986), 155-178.

Institute of Mathematics

Jagiellonian University

Reymonta 4

30-059 Kraków, Poland

E-mail: Maciej.Denkowski@im.uj.edu.pl

Rafal.Pierzchala@im.uj.edu.pl

Received 8.2.2007

and in final form 21.1.2008 